\title{
Rapid Access to Medications for Opioid Use Disorder
}

\author{
Robert A. Kleinman, $\mathrm{MD}^{1,2}$ (1) and Nathaniel P. Morris, $\mathrm{MD}^{3}$ \\ 'Massachusetts General Hospital, Boston, MA, USA; ${ }^{2}$ Harvard Medical School, Boston, MA, USA; ${ }^{3}$ University of California, San Francisco, San \\ Francisco, CA, USA.
}

$\mathrm{J}$ Gen Intern Med 36(11):3557-8

DOI: $10.1007 / \mathrm{s} 11606-021-06850-1$

(c) Society of General Internal Medicine 2021

$\mathrm{O}$ ver 1.5 million people in the USA have an opioid use disorder (OUD), and opioid-involved overdoses are a major cause of death. ${ }^{1}$ Medications for opioid use disorder (MOUD) can substantially reduce morbidity and mortality associated with OUDs, yet just $18 \%$ of people with OUDs in 2019 received these medications during the prior year. ${ }^{1}$ In several situations where timely medical attention is critical, on-demand resources, such as the National Suicide Prevention Lifeline and Poison Control, are available nationwide to rapidly provide consultation or initiate treatment. Expanding readily available access to MOUD is essential, since opioid use, overdose, and other complications often occur at times and places where care is unavailable. ${ }^{2}$

For example, a woman with OUD, lacking housing, and experiencing withdrawal symptoms overnight may find herself at risk for returning to opioid use and potential overdose. If living in Massachusetts, she might search online for treatment options and find a maze of addiction-related websites and hotlines, including the SAMHSA Substance Use Treatment Locator, the National Drug Helpline, and the Massachusetts Substance Use Helpline. Rather than directly providing MOUD, many of these options would refer her to nearby clinics or treatment programs, which also may not answer calls outside business hours or initiate treatment immediately. In a 2020 study, researchers attempted to contact more than 500 buprenorphine providers listed in the SAMHSA Buprenorphine Practitioner Locator, finding just 140 (28\%) had appointments available, ${ }^{3}$ and available appointments may not be for days or weeks following an initial call. Furthermore, some websites and hotlines related to opioid treatment are run by for-profit organizations, which may prioritize other treatment approaches, such as residential programs, over MOUD.

Some researchers and clinicians have argued that people grappling with addiction need better access to on-demand treatment, ${ }^{2}$ and two converging trends suggest that rapid

Received January 13, 2021

Accepted April 22, 2021

Published online May 28, 2021 access to MOUD is now more feasible. First, low-threshold MOUD prescribing is becoming more common among cities in the USA and abroad. ${ }^{4,5}$ People with OUDs often face barriers to routine care, including homelessness, poverty, incarceration, and co-occurring mental disorders. Additional systems-level barriers, including requirements regarding inperson appointments, urine drug screening, and insurance coverage, can further decrease likelihood of treatment with MOUD. Recognizing that the benefits of treatment with MOUD often outweigh the risks, clinicians are striving to overcome these barriers by prescribing MOUD for patients in low-threshold, flexible settings (e.g., walk-in, emergency department, mobile, or bridge clinics), frequently with sameday initiation of treatment and harm reduction interventions. ${ }^{6}$ Research suggests low-threshold MOUD prescribing can help reach marginalized, at-risk patients who otherwise might not receive evidence-based care and connect these patients with longer-term treatment. ${ }^{4,5}$

Second, the COVID-19 pandemic has shifted the ways in which clinicians can offer MOUD. Previously, federal regulations, including those enacted by the Ryan Haight Act, generally prohibited prescribers from initiating treatment with agonist MOUD without an in-person examination. To mitigate the spread of COVID-19, federal officials waived a number of these regulations, allowing prescribers greater flexibility to prescribe MOUD remotely (including video- and telephonebased encounters). At least one bipartisan bill introduced in Congress during 2020 would allow video, though not audio, telehealth buprenorphine prescribing on a permanent basis.

In several jurisdictions, health professionals have already made use of newly flexible telemedicine rules to reduce barriers to buprenorphine treatment for OUD. Clinicians in Rhode Island developed a telephone-based clinic for low-threshold outpatient buprenorphine initiation and connection to ongoing care. $^{7}$ Once connected with this "tele-bridge" clinic, callers could undergo same-day assessments and, if indicated, start MOUD with linkage to longer-term care. ${ }^{7}$ In New York City, a virtual clinic offering telephone- or video-based care was able to initiate 78 patients on buprenorphine and completed 252 patient visits over its first 9 weeks of operation, with 42 (54\%) of patients remaining in care at 8 weeks of follow-up. ${ }^{8}$

By rapidly connecting people with OUDs to clinical evaluation and, when indicated, treatment with MOUD, these emerging, low-barrier and telemedicine-enabled models of care offer considerable promise for shaping the delivery of 
OUD treatment. Low-threshold telephone or video-based clinics may mitigate geographic disparities in access to specialty addiction care and MOUD. In 2018, 40\% of US counties did not have a single provider who could prescribe buprenorphine for OUD. ${ }^{9}$ When prescribed by clinicians through telemedicine visits, buprenorphine and naltrexone can be dispensed by local pharmacies, which are accessible in both urban and rural counties. ${ }^{10}$ Pharmacies may also provide naloxone, safer injection supplies, and other harm reduction interventions. Rapid access to MOUD is key for supporting people with OUDs experiencing transitions in care, for instance upon release from incarceration, as has been the case in New York City. ${ }^{8}$ Beyond providing care to patients with OUDs, rapid access clinics may also provide substance use disorder evaluation and treatment for other substances, such as alcohol or stimulants. ${ }^{5}$ In addition, telemedicineenabled rapid access clinics can offer provider-facing addiction consultation to support health professionals with treatment-related questions.

Despite the potential benefits of rapid access clinics for MOUD prescribing, these models still face limitations. Different patients may benefit from different MOUD and, compared with buprenorphine and naltrexone, methadone remains subject to stricter regulations that may prevent telemedicineenabled clinics from offering this medication. Given shortages of MOUD prescribers, especially in rural regions, rapid access clinics may encounter difficulties in coordinating local followup care and require capacity to provide ongoing treatment for some patients. Difficulty recruiting MOUD prescribers willing to work overnight or on weekends may limit the degree to which rapid access clinics can operate - and therefore potentially connect patients with MOUD - outside usual business hours. Public funding or private donations may be necessary to support care of uninsured or underinsured patients by rapid access clinics. Some policymakers and others in the public may oppose these models of care due to concerns about indiscriminate MOUD prescribing and stigma regarding addiction treatment, which could complicate program funding and approval. When providing telemedicine-based services, rapid access clinics will need to partner with local laboratories for urine drug screening, as well as collaborate with pharmacies and prescription drug monitoring programs, to mitigate risks of medication diversion or misuse. The degree to which stricter regulations on MOUD prescribing might return beyond the COVID-19 pandemic also remains unknown.

Not all patients with OUDs will need or benefit from rapid access clinics for MOUD. Many people with OUDs lack consistent telephone or Internet access, so telephone- or videobased clinics alone may not be enough to meet these patients' needs. Even among individuals with telephone or Internet access, identifying rapid access clinics that provide MOUD may be challenging when searching through the confusing landscape of existing addiction-related websites and hotlines.
Additional strategies, such as regularly updating treatment locator databases with clear information about rapid access clinic options or providing direct access to treatment through a centralized (e.g., state-level or national) website or hotline, may support patients and families navigating these resources. ${ }^{3}$

Further research is needed to compare the effectiveness of traditional approaches to MOUD prescribing with these emerging models of rapid MOUD access. Yet, existing systems for delivering MOUD in the USA are clearly not working - most people with OUDs do not receive treatment, and over 45,000 people are dying from opioid-involved overdoses each year. Opioid addiction is not limited to specific hours or places, and life-saving treatment should not be either.

Corresponding Author: Robert A. Kleinman, MD; Massachusetts General Hospital, Boston, MA, USA

\section{Declarations:}

Conflict of Interest: The authors declare that they do not have a conflict of interest.

\section{REFERENCES}

1. Substance Abuse and Mental Health Services Administration. Key Substance Use and Mental Health Indicators in the United States: Results from the 2019 National Survey on Drug Use and Health [Internet]. Rockville: Center for Behavioral Health Statistics and Quality, Substance Abuse and Mental Health Services Administration; 2020. Available from: https://www.samhsa.gov/data/

2. Wenger LD, Rosenbaum M. Drug treatment on demand-not. J Psychoactive Drugs 1994;26(1):1-11.

3. Flavin L, Malowney M, Patel NA, et al. Availability of Buprenorphine Treatment in the 10 States With the Highest Drug Overdose Death Rates in the United States. Journal of Psychiatric Practice 2020;26(1):17-22.

4. Krawczyk N, Buresh M, Gordon MS, Blue TR, Fingerhood MI, Agus D. Expanding low-threshold buprenorphine to justice-involved individuals through mobile treatment: Addressing a critical care gap. J Subst Abuse Treat 2019;103:1-8.

5. Wiercigroch $\mathbf{D}$, Sheikh $\mathbf{H}$, Hulme $\mathbf{J}$. A rapid access to addiction medicine clinic facilitates treatment of substance use disorder and reduces substance use. Subst Abuse Treat Prev Policy. 2020;15(1):4.

6. Jakubowski A, Fox A. Defining Low-threshold Buprenorphine Treatment. Journal of Addiction Medicine 2020;14(2):95-8.

7. Samuels EA, Clark SA, Wunsch C, et al. Innovation During COVID-19: Improving Addiction Treatment Access. J Addict Med 2020;14(4):e8-e9.

8. Tofighi B, McNeely $\mathbf{J}$, Walzer $\mathbf{D}$, et al. A Telemedicine Buprenorphine Clinic to Serve New York City: Initial Evaluation of the NYC Public Hospital System's Initiative to Expand Treatment Access during the COVID-19 Pandemic. J Addict Med. 2021; Online Ahead of Print. doi: 10.1097/ADM.0000000000000809

9. Office of Inspector General, U.S. Department of Health and Human Services. Geographic Disparities Affect Access to Buprenorphine Services for Opioid Use Disorder. 2020 [cited 2021 Jan 2]. Available from: https:// oig.hhs.gov/oei/reports/oei-12-17-00240.pdf.

10. Kleinman RA. Comparison of Driving Times to Opioid Treatment Programs and Pharmacies in the US. JAMA Psychiatry 2020;77(11):1163-71.

Publisher's Note: Springer Nature remains neutral with regard to jurisdictional claims in published maps and institutional affiliations. 